\title{
Clinical assessment for identifying causes of acute respiratory failure in cancer patients
}

\author{
David Schnell ${ }^{1,2}$, Julien Mayaux ${ }^{1,2}$, Jérôme Lambert ${ }^{2,3}$, Antoine Roux ${ }^{1,2}$, \\ Anne-Sophie Moreau ${ }^{1,2}$, Lara Zafrani ${ }^{1,2}$, Emmanuel Canet ${ }^{1,2}$, Virginie Lemiale ${ }^{1,2}$, \\ Michael Darmon ${ }^{1,2}$ and Élie Azoulay ${ }^{1,2}$
} Affiliations: ${ }^{1}$ Medical Intensive Care Unit, Hôpital Saint-Louis, Paris, ${ }^{2}$ UFR de Médecine, University Paris-7
Paris-Diderot, Paris, ${ }^{3}$ Biostatistics Dept, Hôpital Saint-Louis, Paris, France.

Correspondence: É. Azoulay, Réanimation Médicale, AP-HP, Hôpital Saint-Louis, 1 Avenue Claude Vellefaux, 75010, Paris, France. E-mail: élie.azoulaydasls.aphp.fr

ABSTRACT In cancer patients with acute respiratory failure (ARF), early adequate therapy is associated with better outcomes. We investigated the performance of the DIRECT approach, which uses criteria available at the bedside at admission to the intensive care unit (ICU), to identify causes of ARF in cancer patients.

This cohort study included cancer patients with ARF of determined aetiology. Associations of aetiological groups with the selected criteria were evaluated using correspondence analysis.

424 cancer patients were included: 201 (47\%) with bacterial pneumonia, 131 (31\%) with opportunistic infections and $92(22 \%)$ with noninfectious disorders. Mechanical ventilation (both invasive and noninvasive) was needed in 328 (77\%) patients, treatment for shock in 217 (51\%) patients and dialysis in 82 (19\%) patients. 142 (34\%) patients died in the ICU. Correspondence plots showed that bacterial pneumonia was associated with neutropenia, solid tumour, multiple myeloma, $<3$ days since symptom onset, shock, unilateral crackles and unilateral radiographic pattern. Opportunistic infections were associated with steroids, lymphoproliferative disorders and haematopoietic stem-cell transplantation, whereas noninfectious disorders were associated with acute leukaemia

The selected criteria are strongly associated with causes of ARF in cancer patients and could be used to develop an algorithm for selecting first-line diagnostic investigations and empirical treatments.

@ERSpublications

Clinical assessment using the DIRECT criteria may help identify causes of acute respiratory failure in cancer patients http://ow.ly/kNQZD

For editorial comments see page 299 .

This article has supplementary material available from www.erj.ersjournals.com

Received: Aug 062012 | Accepted after revision: Oct 152012 | First published online: Nov 082012

Support statement: This study was supported by a grant from the Assistance-Publique Hôpitaux de Paris [AOM 04139] and the French Society for Intensive Care Medicine.

Conflict of interest: None declared.

Copyright @ERS 2013 


\section{Introduction}

The number of cancer patients has increased over the last few decades $[1,2]$ as a result of survival gains achieved by intensive anticancer treatments and improvements in supportive care [3-7]. These survival gains have come at the cost of increases in toxic and infectious complications [6-9]. Among these complications, acute respiratory failure (ARF) occurs in up to $30 \%$ of cancer patients and is the leading reason for admission to the intensive care unit (ICU) in this population. ARF in cancer patients still carries a high mortality rate of $50 \%$ overall, with even higher rates in patients needing mechanical ventilation or having no identifiable cause of ARF [10-14]. Fibreoptic bronchoscopy with bronchoalveolar lavage (FOB$\mathrm{BAL}$ ) has long been considered the cornerstone of the aetiological diagnosis [15]. This procedure has been associated with adverse events [16-19], however, especially in the most unstable patients [20-22]. A recent study showed that FOB-BAL performed in the ICU was not associated with an increased rate of mechanical ventilation [14]. Nevertheless, compared to a noninvasive diagnostic strategy, the diagnostic strategy including FOB-BAL did not increase the proportion of patients for whom a cause was identified and it had only a small impact on treatment decisions [14]. The cause of ARF is not always identifiable in cancer patients $[13,14,23]$. Clinicians must be aware that the potential benefit of identifying the cause of ARF must be balanced against the risk of adverse events related to FOB-BAL, especially in hypoxaemic ICU patients $[12,24]$.

The first step in the aetiological diagnosis of ARF in a patient with cancer consists of a systematic clinical evaluation $[14,24]$ aimed at identifying the most likely causes, and therefore, at determining which first-line diagnostic investigations and empirical treatments are most likely to benefit the patient. We have suggested that this evaluation should focus on six factors, which can be listed using the mnemonic DIRECT (table 1): delay since the onset of malignancy or haematopoietic stem-cell transplantation (HSCT), since symptom onset and since the implementation of antibiotics/prophylaxis; pattern of immune deficiency; radiographic appearance; experience and knowledge of the literature; clinical picture; and findings by high-resolution computed tomography (HRCT) of the chest $[12,25]$. The DIRECT approach rests on our clinical experience of the diagnostic management of ARF in cancer patients, but has never been evaluated. It consists of using criteria in each of the six categories listed to identify the most likely causes of ARF and to help clinicians to select which diagnostic investigations are required urgently and to choose appropriate empiric treatments.

The purpose of this study was to obtain preliminary information on the usefulness of the DIRECT approach for identifying the cause of ARF in cancer patients. To this end, we assessed associations between aetiological diagnoses and DIRECT criteria.

\section{Patients and methods}

Patients with cancer and hypoxaemic ARF admitted to our closed ICU in a teaching hospital between November 1, 1997 and October 31, 2008 were identified in our database and included in this retrospective cohort study. The appropriate review board (Comité d'Ethique, Pitié-Salpêtrière University Hospital, Paris, France) approved the study and waived the requirement for informed consent. Patients were excluded if they had no definite aetiological diagnosis for the episode of ARF and if they had a documented co-infection with bacteria and an opportunistic pathogen.

Cancer patients were defined as patients with active haematological malignancies or solid tumours and/or HSCT. ARF was defined by the presence of respiratory distress symptoms or arterial oxygen tension $\left(\mathrm{PaO}_{2}\right)$ on room air $<8 \mathrm{kPa}$ or a need for ventilatory support. Neutropenia was defined as a neutrophil count $<500$ cells $\cdot \mathrm{mm}^{-3}$ within $24 \mathrm{~h}$ after ICU admission [26]. Septic shock was defined as an acute circulatory failure (systolic blood pressure $<90 \mathrm{mmHg}$ or mean blood pressure $<60 \mathrm{mmHg}$ combined with clinical signs of acute circulatory failure (cold extremities, skin mottling, oliguria and mental confusion)) persisting

TABLE 1 DIRECT criteria for identifying the most likely causes of acute respiratory failure in cancer patients $[12,25]$

Delay since malignancy onset or HSCT, since symptom onset and since implementation of antibiotics/ prophylaxis

Pattern of immune deficiency

Radiographic appearance

Experience and knowledge of the literature

Clinical picture (including ongoing chemoprophylaxis and effective antibiotic therapy)

Findings by HRCT

HSCT: haematopoietic stem-cell transplantation; HRCT: high-resolution computed tomography. 
despite adequate fluid resuscitation and requiring treatment with vasopressors. Patient health before ICU admission was assessed using the Karnofsky performance status scale [27].

Microbiologically documented pneumonia was defined as an ARF episode consistent with bacterial infection and identification of pathogenic bacteria in sputum, BAL fluid, or blood cultures. Clinically documented pneumonia was defined as ARF without microbial documentation but consistent with bacterial infection, i.e. displaying at least two of the four following criteria: consolidation on chest radiography; purulent sputum and/or neutrophil alveolitis by BAL fluid analysis; and rapid resolution with antibiotics. Patients with a positive respiratory specimen but no clinical signs of pneumonia and those with signs of pneumonia but nonpathogenic bacteria in the sputum were considered to have colonisation. All other infections required microbial documentation.

Patient management was at the discretion of the attending physicians. The data reported in tables 1, 2 and 3 and in figure 1 were collected for each study patient. The logistic organ dysfunction scores, duration of invasive and noninvasive mechanical ventilation, time spent in the ICU and vital status at ICU and hospital discharge were also collected [28].

All patients were investigated using a previously described diagnostic strategy that relies heavily on noninvasive tests [24]. Most of the patients underwent noninvasive tests to look for infections, such as sputum examination for bacteria, mycobacteria and fungi; induced sputum for Pneumocystis jirovecii pneumonia; serum and blood tests for circulating Aspergillus galactomannan; blood cultures; specific PCR tests for herpes viruses including cytomegalovirus on blood and respiratory samples; PCR tests for $P$. jirovecii on respiratory samples; immunofluorescence staining of respiratory samples to detect antigens of respiratory viruses (including influenza $\mathrm{A}$ and $\mathrm{B}$ viruses, respiratory syncytial virus, parainfluenza viruses and adenoviruses); and urine tests for bacterial antigens. Fibreoptic bronchoscopy with BAL was performed when deemed appropriate by the attending physician. BAL fluid was collected as previously described [24] and was used for bacterial, mycobacterial and fungal cultures; respiratory virus antigen detection by immunofluorescence; and cytological examination. ECG, HRCT and thoracocentesis were performed when deemed appropriate by the attending physician.

In all study patients, the aetiological diagnosis of ARF was established by the investigators based on a review of the patient's medical charts. The diagnoses were based on clinical, radiographic, microbiological and histological findings, according to predefined criteria [10, 23, 29]. The results of noninvasive tests (PCR for $P$. jirovecii and cytomegalovirus, or immunofluorescence for respiratory viruses) were not necessarily considered diagnostic, and they were interpreted according to the degree of clinical and radiological suspicion [14, 30]. For P. jirovecii, a negative PCR test was used to rule out the diagnosis of Pneumocystis pneumonia and a positive test was considered diagnostic only if there were radiographic criteria suggestive of the diagnosis (i.e. bilateral ground-glass opacities without pleural effusion). All other cases were considered to be contamination or airway colonisation.

All invasive fungal infections were diagnosed according to criteria issued by the European Organisation for Research and Treatment of Cancer/Mycosis Study Group [31, 32]. Aetiologies of ARF were further divided into three categories requiring different treatments (i.e. antibacterial agents, antifungal and antiviral agents or steroids and diuretics): bacterial pneumonia (either microbiologically documented or clinically documented); opportunistic pulmonary infection ( $P$. jirovecii pneumonia, pulmonary aspergillosis, viral pneumonia and Toxoplasma gondii pneumonia); and noninfectious lung disorders including pulmonary oedema (with electrocardiographic documentation), pulmonary drug toxicity and pulmonary involvement with the malignancy (carcinomatous lymphangitis, leukostasis and pulmonary leukaemic infiltration, malignant pleural effusion and lung cancer or metastasis).

The study variables were the DIRECT criteria except experience and knowledge of the literature (table 1): delay since onset of symptoms ( $\leqslant 3$ days, $4-7$ days or $>7$ days); pattern of immune deficiency (multiple myeloma, solid tumour, neutropenia, allogeneic HSCT, steroids, acute leukaemia or lymphoproliferative diseases); radiographic appearance (alveolar pattern, unilateral or bilateral pattern, interstitial pattern, cardiomegaly or pleural effusion); clinical picture (fever, bronchial breathing, shock and unilateral or bilateral crackles); and HRCT findings (consolidation, ground-glass opacities, nodules and/or septal lines).

\section{Statistics}

Quantitative parameters are reported as median and interquartile range and qualitative parameters as $\mathrm{n}$ (\%). Categorical variables were compared using the Chi-squared test or Fisher's exact test and continuous variables were compared using the Mann-Whitney U-test or Wilcoxon test, as appropriate. Odds ratios and their $95 \%$ confidence intervals were computed. 
TABLE 2 Baseline characteristics, underlying malignancy, need for life-supporting interventions and outcomes of 424 cancer patients with acute respiratory failure

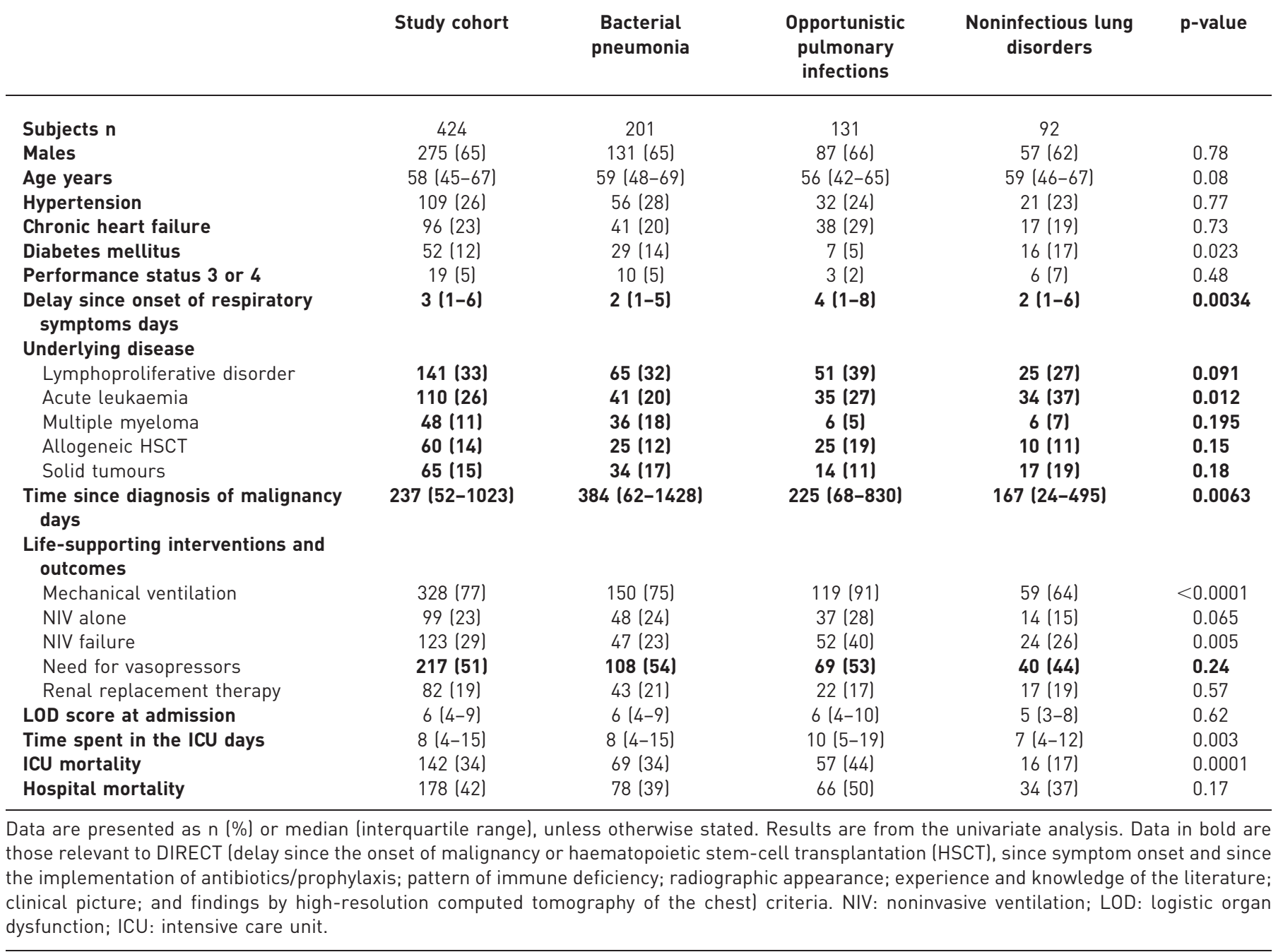

We also used correspondence analysis to investigate the associations between the three above-mentioned diagnostic categories of ARF and DIRECT criteria. Correspondence analysis is a multivariate descriptive statistical technique for analysing two-way contingency tables by measuring the correlations between rows and columns. The distance between row and column points reflects the correlation between two variables: tightly correlated variables are located near each other. Thus, the association between any two variables can be depicted in a two-dimensional plane. This graphical presentation helps to detect unexpected associations between variables. Correspondence analysis provides information similar to that produced by factor analysis, although it is a nonparametric method and thus makes no assumption about distribution. The overall Chi-squared value of the contingency table reflects the degree of departure from a purely random distribution of the distances between variables (here, diagnostic categories and DIRECT criteria). When no relationship exists, Chi squared equals zero. Correspondence analysis partitions Chi-square into two orthogonal dimensions. The advantage of this multivariate approach is that it analyses the data globally and takes into account the correlations among the variables, thus reducing bias in the results. The final goal of correspondence analysis is to obtain meaningful, albeit subjective, interpretations of the two extracted dimensions.

p-values $<0.05$ were considered significant. Statistical analyses were performed using Statview 5.0 (SAS Institute, Cary, NC, USA).

\section{Results}

We included 424 cancer patients admitted to our ICU with ARF due to known causes during the study period. During the same period, 122 cancer patients were admitted with ARF of unknown cause, 97 patients 


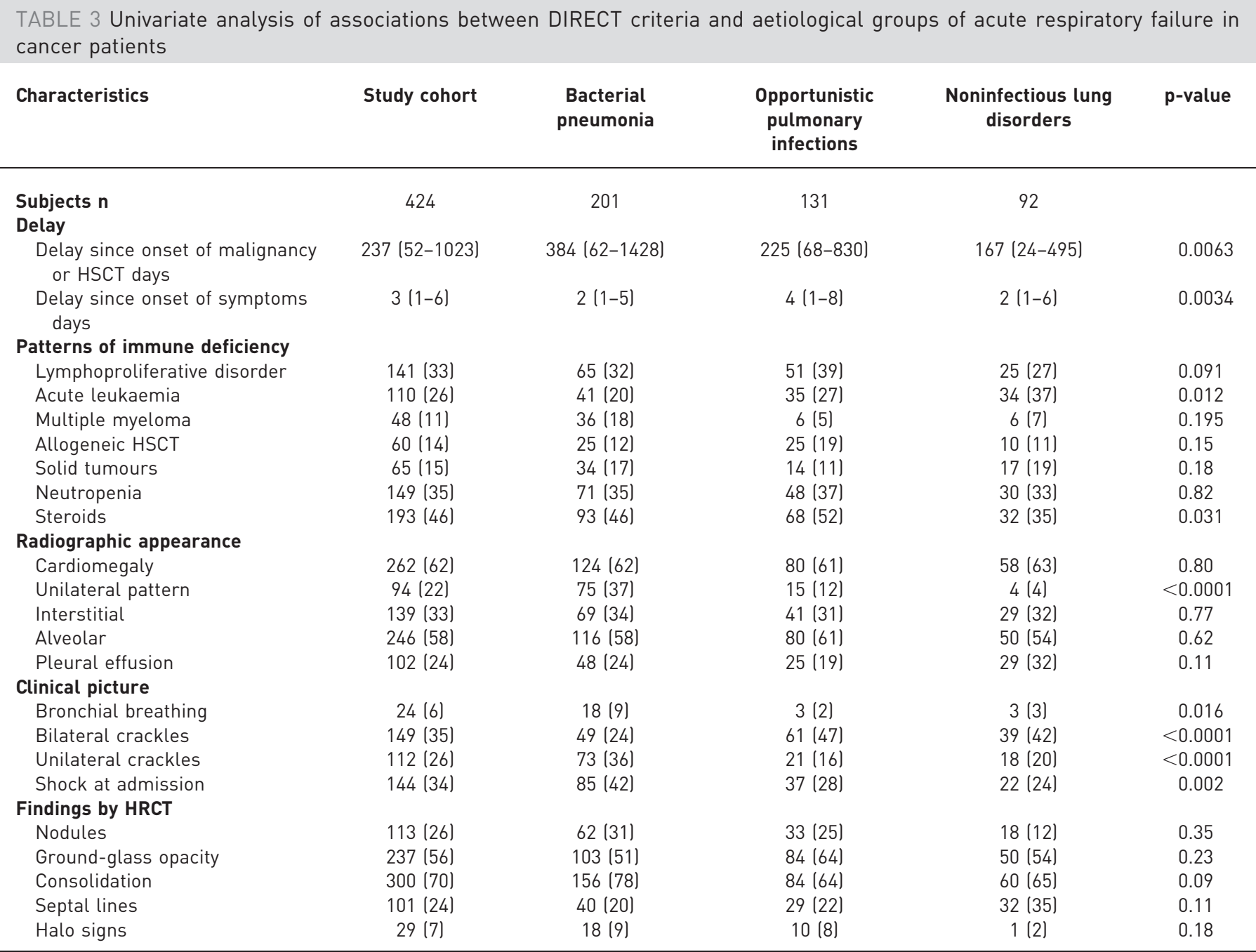

Data are presented as $\mathrm{n}(\%)$ or median (interquartile range), unless otherwise stated. Results are from univariate analysis. DIRECT: delay since the onset of malignancy or haematopoietic stem-cell transplantation (HSCT), since symptom onset and since the implementation of antibiotics/ prophylaxis; pattern of immune deficiency; radiographic appearance; experience and knowledge of the literature; clinical picture; and findings by high-resolution computed tomography (HRCT) of the chest.

were admitted with ARF due to suspected causes (e.g. diffuse alveolar haemorrhage) or miscellaneous causes (e.g. pulmonary embolism) and 11 patients were admitted with a documented co-infection with bacteria and opportunistic pathogens; these 230 patients were not included. Table 2 lists the main characteristics of the patients. All patients were admitted to the ICU for ARF and 144 (34\%) also had shock at admission. At ICU admission, 149 (35\%) patients had neutropenia, 193 (46\%) were receiving steroids and 292 (69\%) were on antibiotics. Table 2 reports the life-supporting interventions and outcomes.

The diagnostic investigations for ARF included FOB-BAL in 277 (65\%) patients and HRCT in 208 (49\%). The main causes of ARF were bacterial infections in 201 (47\%) patients, opportunistic pulmonary infections in $131(31 \%)$ patients and noninfectious lung disorders in $92(22 \%)$ patients. Bacterial infections were microbiologically documented in $80(40 \%)$ out of 201 patients and clinically documented in $121(60 \%)$ out of 201. Online supplementary table E1 lists the documented pathogens. The 131 patients with opportunistic pulmonary infections had invasive pulmonary aspergillosis $(n=41 ; 31 \%)$, respiratory viral infections $(\mathrm{n}=37 ; 28 \%)$, P. jirovecii pneumonia $(\mathrm{n}=36 ; 27.5 \%)$, tuberculosis $(\mathrm{n}=6 ; 5 \%)$, mucormycosis $(\mathrm{n}=5 ; 4.5 \%)$, cytomegalovirus infection $(n=2 ; 1.5 \%)$, fusariosis $(n=2 ; 1.5 \%)$, Scedosporium sp. infection $(n=1 ; 1 \%)$, and T. gondii infection $(\mathrm{n}=1 ; 1 \%)$. The diagnoses in the 92 patients with noninfectious lung disorders were pulmonary oedema $(n=45 ; 49 \%)$, lung cancer or metastasis $(n=45 ; 49 \%)$ and pulmonary drug toxicity $(\mathrm{n}=2 ; 2 \%$, due to bleomycin and rituximab). Neither patient with drug toxicity underwent lung biopsy. Table 2 compares the three diagnostic groups. The prevalence of neutropenia was similar in the three 

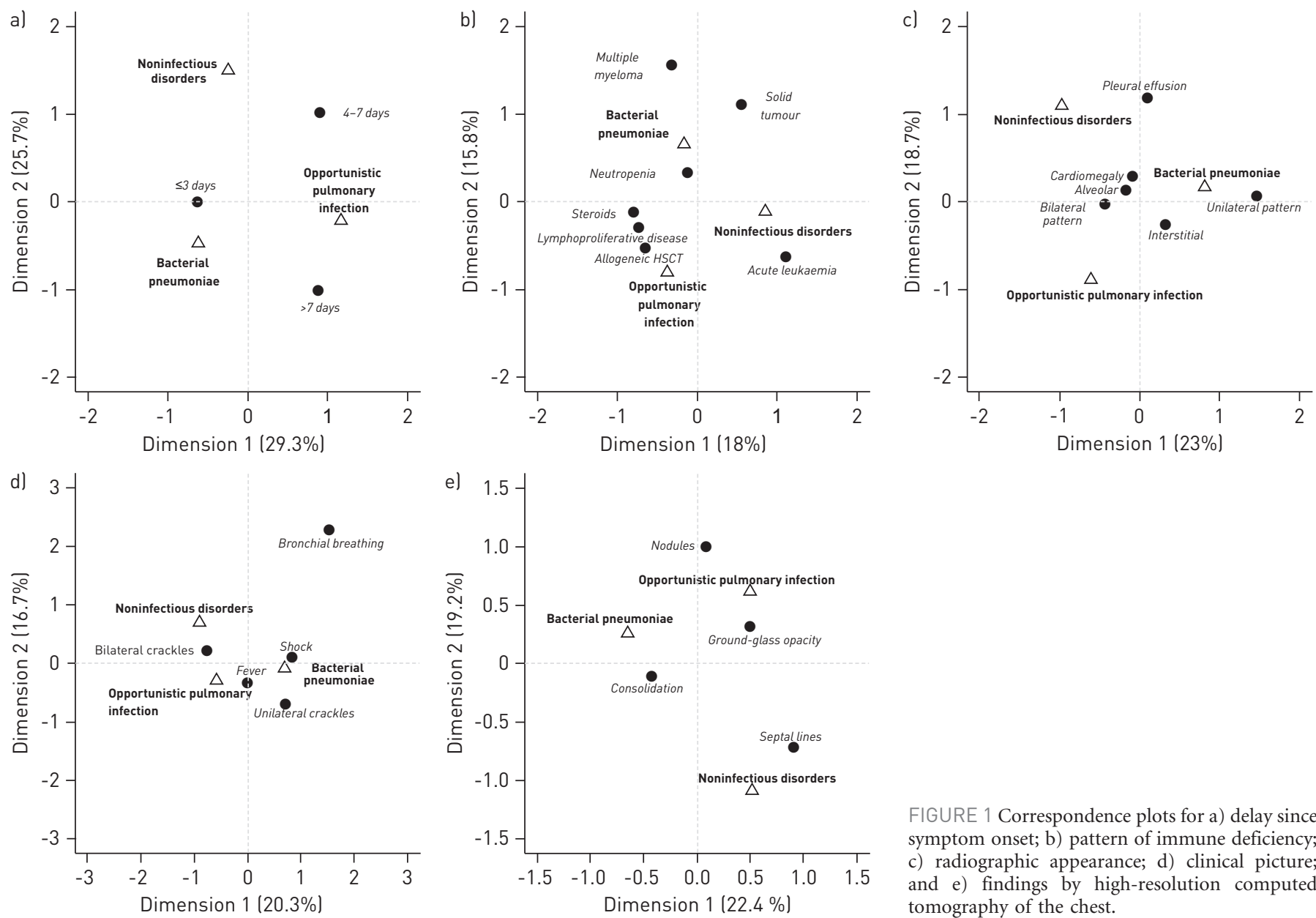

FIGURE 1 Correspondence plots for a) delay since symptom onset; b) pattern of immune deficiency; c) radiographic appearance; d) clinical picture; and e) findings by high-resolution computed tomography of the chest.

groups (35\% in patients with bacterial pneumonia, 37\% in those with opportunistic pulmonary infections and $33 \%$ in those with noninfectious lung disorders; $\mathrm{p}=0.82$ ). Patients with noninfectious lung disorders were less often on steroids at ICU admission (35\% versus $46 \%$ in bacterial infections and $52 \%$ in opportunistic pulmonary infections; $\mathrm{p}=0.031)$ and those with bacterial pneumonia or opportunistic pulmonary infections more often received antibiotics before ICU admission (68\% and 83\%, respectively, versus $51 \%, p=0.002$ ). Finally, patients with bacterial pneumonia more often had shock at admission ( $42 \%$ versus $28 \%$ in opportunistic pulmonary infection and $24 \%$ in noninfectious lung disorders; $\mathrm{p}=0.002)$.

Table 3 compares DIRECT criteria in the three diagnostic groups. Figure 1a shows the results of the correspondence analysis for delay since symptom onset. Dimension 1 (horizontal axis) explained 29.3\% and dimension 2 (vertical axis) $25.7 \%$ of the Chi-squared value. The correspondence plot shows that patients with bacterial pneumonia more often had delays of $<3$ days since symptom onset. Figure $1 \mathrm{~b}$ displays the correspondence analysis for the pattern of immune deficiency. Dimension 1 (horizontal axis) explained $18 \%$ and dimension 2 (vertical axis) $15.8 \%$ of the Chi-squared value. The plot indicates associations of neutropenia, solid tumour and multiple myeloma with bacterial pneumonia and associations of steroids, lymphoproliferative disorders and HSCT with opportunistic pulmonary infection. Acute leukaemia was associated with noninfectious lung disorders. Figure 1c shows the correspondence analysis for radiographic appearance. Dimension 1 (horizontal axis) explained 23\% and dimension 2 (vertical axis) 18.7\% of the Chisquared value. The plot shows a link between unilateral radiographic changes and bacterial infections. Figure 1d displays the correspondence analysis for the clinical picture. Dimension 1 (horizontal axis) explained $20.3 \%$ and dimension 2 (vertical axis) $16.7 \%$ of the Chi-squared value. Bacterial pneumonia was closely linked to shock at ICU admission and less closely linked to unilateral crackles. Finally, figure 1e shows the correspondence analysis for HRCT findings. Dimension 1 (horizontal axis) explained $22.4 \%$ and dimension 2 (vertical axis) 19.2\% of the Chi-squared value. The plot shows a link between unilateral HRCT changes and bacterial pneumonia. 


\section{Discussion}

Using an original methodology in a cohort of ICU patients, we investigated the usefulness of DIRECT criteria for the aetiological diagnosis of ARF in cancer patients. Strong links were documented between the three aetiological groups and DIRECT criteria.

Acute respiratory failure is a common and life-threatening event in cancer patients. It represents the leading reason for ICU admission in these patients, and intensivists will be increasingly asked to managed these patients given growing incidence of cancer [1,2] and the improved survival of cancer patients [3-9]. This condition still carries a mortality rate of $\sim 50 \%$ overall, with even higher rates occurring in patients who require mechanical ventilation [10-14]. Studies conducted in the past decade have established that mortality in the ICU is no longer linked to the characteristics of the underlying disease but depends instead on the severity and reversibility of the ARF event $[12,33]$. In particular, mortality is higher when investigations fail to identify the cause of $\operatorname{ARF}[10,11,13,34]$. Thus, rather than relying on broad-spectrum empirical treatments, improving the diagnostic strategy is crucial to increase survival rates [12, 24].

Based on our experience with cancer patients admitted to the ICU, we have suggested six categories of criteria for identifying the cause of ARF, which can be conveniently listed using the mnemonic DIRECT (table 1) [12, 24, 25]. DIRECT may assist in the management of ARF in cancer patients by focusing the diagnostic investigations on the most likely causes and guiding empirical treatments [12, 24, 25, 35]. To assess the usefulness of DIRECT, we used correspondence analysis to investigate associations between three diagnostic categories of ARF having different therapeutic requirements and the DIRECT criteria. This descriptive nonparametric statistical method with a graphical display of the results showed that bacterial pneumonia was associated with rapid-onset ARF, typically occurring in patients with neutropenia, multiple myeloma or solid tumour, and was responsible for shock, unilateral crackles and unilateral consolidations. However, these associations should be interpreted with great caution: in our experience, bacterial pneumonia is the main cause of ARF in patients with neutropenia, multiple myeloma or solid tumour, but can occur in patients with nearly all types of malignancy. We also found that opportunistic pulmonary infections occurred chiefly in patients with lymphoproliferative disorders, HSCT or steroid therapy. These associations reflect the predominance among opportunistic pulmonary infections of invasive pulmonary aspergillosis, seen mainly in HSCT recipients [31], and pneumocystis pneumonia, a disease associated with lymphocyte dysfunction [36]. Finally, noninfectious lung disorders were associated with acute leukaemia. This association probably reflects the high proportion of patients with pulmonary leukostasis in the group with noninfectious lung disorders.

Given the multiplicity of aetiological diagnoses and of DIRECT criteria, a more detailed search for associations seems difficult because the number of comparisons would be too large for statistical validity. As stated above, correspondence analysis is useful to detect unexpected relations between variables. The main limitation of the method is that an observed association only makes sense when interpreted in the light of its clinical and pathophysiological plausibility. In our study cohort, univariate analysis was less effective in detecting associations between aetiologies of ARF and underlying malignancies. Nevertheless, not all aetiologies of ARF occur in all malignancies. This point underlines the usefulness of correspondence analysis. Correspondence analysis could be used to develop a detailed clinical description of each ARF aetiology in cancer patients.

Our study has several limitations. First, each item of the DIRECT approach was studied separately. The main limitation of the present study is that it was not designed to evaluate the DIRECT approach as a whole and whether it may be useful to separate patients requiring different investigations and treatments. Second, patient management was at the discretion of the physicians in charge, and all diagnostic investigations were not performed routinely in all patients. However, this study design does reflect everyday clinical practice. Third, patients in whom the cause of ARF was not identified were not included in this preliminary study. Inclusion of these patients would probably have modified our results, and further studies are needed to investigate the usefulness of DIRECT criteria in unselected cancer patients with ARF. Also, the exclusion of patients who had co-infection with bacterial and opportunistic pathogen probably impacted our results. Fourth, associations detected by correspondence analysis must be interpreted with caution, in the light of pathophysiological and clinical factors. Fifth, our study did not evaluate the "experience and knowledge of the literature" item, which can be expected to play an important role in the effectiveness of DIRECT. Finally, our results were obtained in cancer patients and may not apply to patients with other causes of immunodeficiency.

In this cohort study, we used an original methodology to identify clear associations between groups of aetiological diagnoses and DIRECT criteria. These preliminary results suggest that an algorithm based on DIRECT criteria may be useful in clinical practice by narrowing the spectrum of possible aetiologies, 
thereby guiding urgent investigations and empirical treatments. Our promising results encourage further studies investigating the ability of DIRECT criteria to improve treatment decisions and patient survival.

\section{Acknowledgements}

The authors are indebted to A. Wolfe (Issy les Moulineaux, France) for helping to prepare the manuscript.

\section{References}

1 Coleman MP, Quaresma M, Berrino F, et al. Cancer survival in five continents: a worldwide population-based study (CONCORD). Lancet Oncol 2008; 9: 730-756.

Jemal A, Siegel R, Ward E, et al. Cancer statistics, 2008. CA Cancer J Clin 2008; 58: 71-96.

Azoulay E, Alberti C, Bornstain C, et al. Improved survival in cancer patients requiring mechanical ventilatory support: impact of noninvasive mechanical ventilatory support. Crit Care Med 2001; 29: 519-525.

4 Darmon M, Thiery G, Ciroldi M, et al. Should dialysis be offered to cancer patients with acute kidney injury? Intensive Care Med 2007; 33: 765-772.

5 Brenner H, Gondos A, Arndt V. Recent major progress in long-term cancer patient survival disclosed by modeled period analysis. J Clin Oncol 2007; 25: 3274-3280.

6 Pfreundschuh M, Trümper L, Osterborg A, et al. CHOP-like chemotherapy plus rituximab versus CHOP-like chemotherapy alone in young patients with good-prognosis diffuse large-B-cell lymphoma: a randomised controlled trial by the MabThera International Trial (MInT) Group. Lancet Oncol 2006; 7: 379-391.

7 Coiffier B, Lepage E, Briere J, et al. CHOP chemotherapy plus rituximab compared with CHOP alone in elderly patients with diffuse large-B-cell lymphoma. N Engl J Med 2002; 346: 235-242.

8 Bodey GP. Unusual presentations of infection in neutropenic patients. Int J Antimicrob Agents 2000; 16: 93-95.

9 Richardson PG, Sonneveld P, Schuster MW, et al. Bortezomib or high-dose dexamethasone for relapsed multiple myeloma. N Engl J Med 2005; 352: 2487-2498.

10 Azoulay E, Thiéry G, Chevret S, et al. The prognosis of acute respiratory failure in critically ill cancer patients. Medicine (Baltimore) 2004; 83: 360-370.

11 Hilbert G, Gruson D, Vargas F, et al. Noninvasive ventilation in immunosuppressed patients with pulmonary infiltrates, fever, and acute respiratory failure. N Engl J Med 2001; 344: 481-487.

12 Azoulay E, Schlemmer B. Diagnostic strategy in cancer patients with acute respiratory failure. Intensive Care Med 2006; 32: 808-822.

13 Azoulay E, Mokart D, Rabbat A, et al. Diagnostic bronchoscopy in hematology and oncology patients with acute respiratory failure: prospective multicenter data. Crit Care Med 2008; 36: 100-107.

14 Azoulay E, Mokart D, Lambert J, et al. Diagnostic strategy for hematology and oncology patients with acute respiratory failure: randomized controlled trial. Am J Respir Crit Care Med 2010; 182: 1038-1046.

15 Mayaud C, Cadranel J. A persistent challenge: the diagnosis of respiratory disease in the non-AIDS immunocompromised host. Thorax 2000; 55: 511-517.

16 Patel NR, Lee PS, Kim JH, et al. The influence of diagnostic bronchoscopy on clinical outcomes comparing adult autologous and allogeneic bone marrow transplant patients. Chest 2005; 127: 1388-1396.

17 Davies L, Mister R, Spence DP, et al. Cardiovascular consequences of fibreoptic bronchoscopy. Eur Respir J 1997; 10: 695-698.

18 Jones AM, O’Driscoll R. Do all patients require supplemental oxygen during flexible bronchoscopy? Chest 2001; 119: 1906-1909.

19 Weiss SM, Hert RC, Gianola FJ, et al. Complications of fiberoptic bronchoscopy in thrombocytopenic patients. Chest 1993; 104: 1025-1028.

20 Bauer TT, Torres A, Ewig S, et al. Effects of bronchoalveolar lavage volume on arterial oxygenation in mechanically ventilated patients with pneumonia. Intensive Care Med 2001; 27: 384-393.

21 Verra F, Hmouda H, Rauss A, et al. Bronchoalveolar lavage in immunocompromised patients. Clinical and functional consequences. Chest 1992; 101: 1215-1220.

22 Du Rand IA, Barber PV, Goldring J, et al. Summary of the British Thoracic Society guidelines for advanced diagnostic and therapeutic flexible bronchoscopy in adults. Thorax 2011; 66: 1014-1015.

23 Rañó A, Agustí C, Jimenez P, et al. Pulmonary infiltrates in non-HIV immunocompromised patients: a diagnostic approach using non-invasive and bronchoscopic procedures. Thorax 2001; 56: 379-387.

24 Azoulay E, de Miranda S, Bèle N, et al. Stratégie diagnostique dans l'insuffisance respiratoire aiguë des patients d'oncohématologie. [Diagnostic strategy for acute respiratory failure in patients with haematological malignancy]. Rev Mal Respir 2008; 25: 433-449.

25 Camous L, Lemiale V, Kouatchet A, et al. Minimally invasive diagnostic strategy in immunocompromised patients with pulmonary infiltrates. In: Azoulay E, ed. Pulmonary Involvement in Patients with Hematological Malignancies. Berlin, Springer-Verlag, 2011; pp. 175-190.

26 Freifeld AG, Bow EJ, Sepkowitz KA, et al. Clinical practice guideline for the use of antimicrobial agents in neutropenic patients with cancer: 2010 update by the Infectious Diseases Society of America. Clin Infect Dis 2011; 52: e56-e93.

27 Karnofsky DA, Abelmann WH, Craver LF. The use of the nitrogen mustards in the palliative treatment of carcinoma. Cancer 1948; 1: 634-656.

28 Le Gall JR, Klar J, Lemeshow S, et al. The Logistic Organ Dysfunction system. A new way to assess organ dysfunction in the intensive care unit. JAMA 1996; 276: 802-810.

29 Afessa B, Tefferi A, Litzow MR, et al. Outcome of diffuse alveolar hemorrhage in hematopoietic stem cell transplant recipients. Am J Respir Crit Care Med 2002; 166: 1364-1368.

30 Gentile G, Donati PP, Capobianchi A, et al. Evaluation of a score system for the severity and outcome of cytomegalovirus interstitial pneumonia in allogeneic bone marrow recipients. J Infect 1997; 35: 117-123.

31 Ascioglu S, Rex JH, de Pauw B, et al. Defining opportunistic invasive fungal infections in immunocompromised patients with cancer and hematopoietic stem cell transplants: an international consensus. Clin Infect Dis 2002; 34: $7-14$. 
32 Azoulay E, Mayaud C. Pnemopathie à Candida: mythe ou réalité? [Candida pneumopathy: fact or fiction?]. Rev Pneumol Clin 1999; 55: 349-351.

33 Azoulay E, Soares M, Darmon M, et al. Intensive care of the cancer patient: recent achievements and remaining challenges. Ann Intensive Care 2011; $1: 5$.

34 Gruson D, Hilbert G, Valentino R, et al. Utility of fiberoptic bronchoscopy in neutropenic patients admitted to the intensive care unit with pulmonary infiltrates. Crit Care Med 2000; 28: 2224-2230.

35 Lecuyer L, Chevret S, Thiery G, et al. The ICU trial: a new admission policy for cancer patients requiring mechanical ventilation. Crit Care Med 2007; 35: 808-814.

36 Bollée G, Sarfati C, Thiery G, et al. Clinical picture of Pneumocystis jiroveci pneumonia in cancer patients. Chest 2007; 132: 1305-1310. 